\title{
The mean free path for electron conduction in metallic fullerenes
}

\author{
O. Gunnarsson and J.E. Han \\ Max-Planck-Institut für Festkörperforschung, D-70506 Stuttgart, Germany
}

The electrical resistivity $\rho$ of a metal is usually interpreted in terms of the average distance $l$ an electron travels before it is scattered. As the temperature $T$ is raised, $\rho$ is increased and the apparent mean free path $l$ is correspondingly reduced. In this semi-classical picture $l$ cannot be (much) shorter than the distance $d$ between two atoms. This has been confirmed for many systems and was considered a universal behaviour.t. Recently, some apparent exceptions were found, e.g., alkalidoped Fullerenes ${ }^{3} U_{\text {and }}$ high temperature superconductors. These systems could, however, be in exotic states where only a small fraction of the conduction electrons contribute to the conductivity, and $l$ would then have to be correspondingly larger to explain the observed $\rho$. Here we report on an essentially exact calculation for a model of alkali-doped Fullerenes, where the electrons are scattered by intramolecular vibrations. The resistivity at large $T$ corresponds to $l \ll d$, demonstrating that there is no fundamental principle of the type $l>d$. At large $T$ the semi-classical picture breaks down and the electrons cannot be described as quasi-particles.

We have also calculated the resistivity due to electronelectron scattering for a half-filled Hubbard model. In this case the resistivity saturates and $l$ is not very much smaller than $d$. This difference is traced to the difference between bosons and fermions. The resistivity is often calculated using the Boltzmann equation. Although this equation is usually derived semi-classically, assuming $l \gg d$, in our model for electron-vibration scattering it does not break down qualitatively at large $T$, where $l \ll d$. For small $T$ the calculated $\rho$ due to electronvibration scattering has a linear dependence on $T$ and a strong dependence on the pressure, in agreement with experiment 8

For $\mathrm{A}_{3} \mathrm{C}_{60}(\mathrm{~A}=\mathrm{K}, \mathrm{Rb})$ at $T \sim 500 \mathrm{~K}$, the resistivity is $\rho \sim 2-5 \mathrm{~m} \Omega \mathrm{cm}$. Assuming a spherical Fermi surface and three conduction electrons per site, 3 we find that $l \sim 1-2$ $\AA$ is almost an order of magnitude smaller than the separation $d=10 \AA$ of the $\mathrm{C}_{60}$ molecules. The experimental data have substantial uncertainties, but this is unlikely to influence the qualitative discussion of $l$. Different experimental methods (direct and optical) for different types of samples (thin films and doped single crystals) all suggest that $l \ll d$ for large $T$. It has been suggested that the large $\rho$ is due to the electrons being scattered inside the molecules. This would imply scattering between bands separated by at least $1 \mathrm{eV}$, and it plays a fairly small role for experimentaf $T$. In addition, we find that this interband scattering $\mathrm{B}$ reduces the resistivity by providing an additional channel for conduction.

The conduction in $\mathrm{A}_{3} \mathrm{C}_{60}$ takes place in a partly filled $t_{1 u}$ band. The $T$-dependent part of the resistivity is as- sumed to be due to scattering against phonons (vibrations) with $\mathrm{H}_{g}$ symmetry. We therefore consider a model with a three-fold degenerate $t_{1 u}$ level and a five-fold degenerate $\mathrm{H}_{g}$ Jahn-Teller phonon on each molecule, the hopping between the molecules and the coupling between the electrons and the phonons. The hopping takes into account 10 the orientational disorder of the molecules. 11 The one-particle band width is $W=0.6 \mathrm{eV}$ and the phonon frequency is $\omega_{p h}$. The coupling is determined by the dimensionless $\lambda=(5 / 3) N(0) g^{2} / \omega_{p h}$, where $N(0)$ is the density of states per spin, $g$ is the electron-phonon coupling constant and $\omega_{p h}$ is the phonon frequency.

We perform a finite temperature $\mathrm{QMC}$ calculation, 12 treating the phonons quantum mechanically. We calculate the current-current correlation function for imaginary times and make a transformatipn to real frequencies, using a maximum entropy method. 13 The QMC method has no "sign-problem", and the resistivity of the model can be calculated essentially exactly down to quite small $T$.

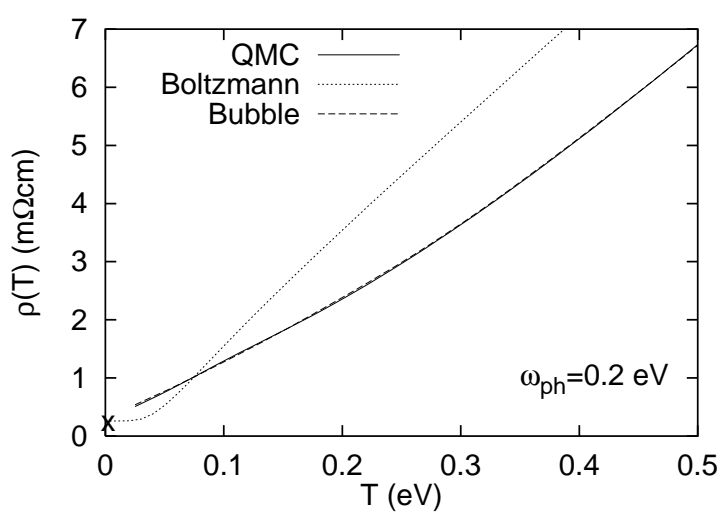

FIG. 1. The resistivity as a function of $T$. Results of the full QMC calculation, the Boltzmann equation (Bloch-Grüneisen) and the bubble diagram are shown. The symbol $\times$ shows the $\rho(T=0)$ due to the orientational disorder. The figure illustrates that $\rho$ can become extremely large, that the bubble calculation is quite accurate and that there is no qualitative break-down of the Boltzmann equation at high $T$.

Fig. 11 shows the resistivity for a cluster of $48 \mathrm{C}_{60}$ molecules for $\lambda=0.5$ and $\omega_{p h}=0.2 \mathrm{eV}$. The QMC calculation (full line) shows that the resistivity can become very large, corresponding to $l \sim 0.7 \AA$ at $T=0.5 \mathrm{eV}$. By also considering an unrealisticly large $T$, we emphasise the lack of a limitation of the type $l>d$. Then there cannot be any general principle requiring $l>d$, because such a principle would then also apply to the present model. Qualitatively similar result were obtained 
by Millis gt al. 14 using the dynamical mean-field theory (DMFT) 15 and assuming classical phonons. Their calculation, however, does not prove that $l \ll d$ is possible, since it involves approximations. The moderate differences to our results are probably due to different models and their use of classical phonons.

While the QMC calculation is essentially exact, it is hard to interpret the results. For this purpose we use a diagrammatic approach. The idea is to simplify the diagrams as far as possible without loosing the qualitative agreement with the QMC results. These diagrams are then studied to obtain a qualitative understanding of the results. This approach (Kubo formalism) requires the calculation of a bubble diagram including vertex corrections (see Fig. 2a). We neglect the vertex and calculate the bubble using the electron Green's function from the QMC calculation. The resulting resistivity (dashed line in Fig. (1) is practically identical to the QMC result, justifying the neglect of vertex corfections for the present model. It was shown by Holstein 16 that in the limit of a broad electronic band, all vertex corrections except ladder diagrams can be neglected and that a Boltzmann equation can be derived. Holstein's derivation is not valid for the narrow band considered here, but our calculations show that his arguments are still rather accurate. For our model with a q-independent electron-phonon coupling, even the ladder diagrams can be neglected. Essentially following Holstein we obtain approximately a Boltzmann like conductivity

$$
\sigma(T) \sim \int d \omega N(\omega)\left(-\frac{d f(\omega)}{d \omega}\right) \frac{1}{\operatorname{Im} \Sigma(\omega)}\left|j_{k}\right|_{\varepsilon_{k}=\omega}^{2},
$$

where $N(\omega)$ is the density of states, $f$ is the Fermi function, $\Sigma(\omega)$ is the electron self-energy, $j_{k}$ is the current matrix element for a state with the label $k$ and the energy $\varepsilon_{k}$. We interpret $\operatorname{Im} \Sigma$ as the inverse of the relaxation time. For a large $T, \operatorname{Im} \Sigma$ becomes comparable to or larger than the one-particle band width. Since $\operatorname{Im} \Sigma$, is related to the inverse life-time of the quasi-particles, this means that the life-times become so short that the concept of a quasi-particle breaks down at large $T$.

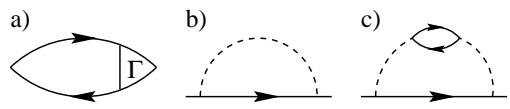

FIG. 2. Relevant diagrams. The current-current response function (a) and two approximations to the electron self-energy ((b) and (c)) are shown. The full and dashed lines represent electron and phonon Green's functions. Self-consistent Green's functions are used in (a) but not in (b) or $(\mathrm{c})$.

The resistivity thus depends crucially on $\Sigma$. To understand its behavior, we consider the diagram in Fig. $2 \mathrm{~b}$ calculated with bare Green's functions and for simplicity neglecting the orbital degeneracy

$$
\Sigma^{(1)}(\mathbf{k}, \omega)
$$

$$
=g^{2} \sum_{\mathbf{q}}\left[\frac{n_{B}\left(\omega_{p h}\right)+1-f\left(\varepsilon_{\mathbf{q}}\right)}{\omega-\omega_{p h}-\varepsilon_{\mathbf{q}}}+\frac{n_{B}\left(\omega_{p h}\right)+f\left(\varepsilon_{\mathbf{q}}\right)}{\omega+\omega_{p h}-\varepsilon_{\mathbf{q}}}\right]
$$

where

$$
n_{B}\left(\omega_{p h}\right)=\frac{1}{e^{\omega_{p h} / T}-1} \quad \longrightarrow \rightarrow \infty \frac{T}{\omega_{p h}}
$$

is the Bose occupation number. For large $T, n_{B}$ becomes large, leading to a large $\operatorname{Im} \Sigma$, a small $\sigma$ and a large $\rho$. The Bose nature of the phonons is therefore of crucial importance for our result.

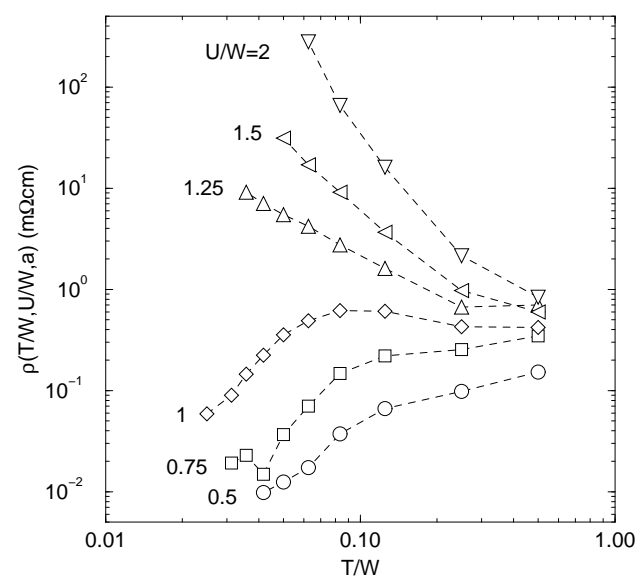

FIG. 3. The resistivity of the nondegenerate Hubbard model. Results are shown for different values of the ratio between the Coulomb repulsion $U$ and the band width $W$. The figure illustrates how the resistivity saturates for large $T$ in the electron-electron scattering model.

This is further illustrated by comparing with the resistivity due to the electron-electron scattering. Using the DMFT we have calculated the resistivity for a nondegenerate Hubbard model on a Bethe lattice with $d=1$ $\AA$. We focus on the half-filled case, which is relevant for $\mathrm{A}_{3} \mathrm{C}_{60}$, and we do not consider the case of a doped Mott insulator 17 The impurity problem is solved with a QMC method. Fig. 3 shows $\rho(T)$ for different values of the onsite Coulomb interaction $U$. For $U / W<1$ the system is a metal and $\rho(T)$ grows with $T$, while for $U / W>1$ it is an insulator and $\rho(T)$ decreases with $T$. The important observation is that in the metallic case $\rho(T)$ saturates at $\rho \sim 0.4 \mathrm{~m} \Omega \mathrm{cm}$, which corresponds to $l / d \sim 1 / 3$. Thus, in contrast to the electron-phonon scattering case, $l$ is not very much smaller than $d$ in the metallic state of this model and within DMFT.

To understand this, we study the electron self-energy $\Sigma$ to second order in $U$, since $\Sigma$ determines $\rho$ in the DMFT. For low $T$, there is little scattering due to the small phase space available, as controlled by the Fermi functions. As $T$ increases, the available phase space grows and $\rho$ increases. However, for large $T, \rho$ essentially saturates, since the Fermi functions approach a constant value. This is in strong contrast to the Bose occupation 
numbers (Eq. (3)), which keep increasing with $T$. The qualitative difference between the two models for large $T$ can then be traced to the difference between fermions and bosons.

We now address the validity of the Boltzmann equation for the case of the electron-phonon scattering in view of $l \ll d$. We have calculated the resistivity using the Ziman version of the Bloch-Grüneisen solution of the Boltzmann equation 18 and added the resistivity due to the orientational disorder as a $T$-independent contribution. 19 Using the plasma frequency $\omega_{p}=1.2 \mathrm{eV}$, we obtain the dotted line in Fig. 11. The Boltzmann result is larger than the QMC result for large $T$, but there is no qualitative break down of the Boltzmann equation, although $l \ll d$ and the quasi-particle concept is not applicable. The justification for the Boltzmann equation in this limit is not the semi-classical derivation, but the (approximate) derivation from the full quantum mechanical Kubo formulation (Eq. (ii)). The proper language is not in terms of a very short mean free path, but in terms of a very broad spectral function ( $\operatorname{Im} \Sigma$ large), as discussed above.

The QMC calculation gives an approximately linear $T$ dependence. This agrees with the experimental result that $\rho$ is linear down to about $100-200 \mathrm{~K} .8$ although the linearity is not as pronounced as for some high temperature superconductors. The result may seem surprising, since at small $T$ the probability of exciting finite energy phonons is exponentially small as is the contribution to $\rho$. Calculating the bubble diagram in Fig. a using electron Green's functions with the self-energy in Fig. $2 \mathrm{~b}$ indeed gives an exponential behaviour. The use of the QMC Green's function, however, gives a linear behavior, in agreement with the full QMC calculation. The reason is that the QMC Green's function also involves processes like in Fig. 2c, where a virtual phonon is created followed by the decay of this phonon into an electron-hole pair.20 The excitation energy of such a pair can be arbitrarily small, leading to a quadratic $T$-dependence for $\rho 20$ In our model this goes over in an approximately linear behavior already for very small $T$.

Calculations for different values of $\lambda$ show a strong $\lambda$ dependence for small $T$. This is in agreement with the strong pressure dependence observed experimentally, since the application of pressure increases the band width and reduces $N(0)$ and thereby $\lambda$. For $\lambda \sim 1$ there is a transition to an insulating state.

Our use of a fairly realistic model with a three-fold degenerate electronic level and a five-fold degenerate phonon is not crucial for the qualitative behaviour of $\rho(T)$, like $l \ll d$ for large $T$. An essential feature is, however, the use of intramolecular phonons acting on a narrow band. The phonon induced shifts $(\sim T)$ of the $t_{1 u}$ levels can be substantial compared with the small band width. It would be interesting to perform calculations for a model where the phonons couple to the hopping matrix elements and where interband transitions become important for large $T$. Such a model would be more relevant for, e.g., transition metal compounds, where the resistivity typically saturates when $l \sim d$.

${ }^{1}$ Fisk, Z. \& Webb, W.B. Saturation of the high-temperature normal-state electrical resistivity of superconductors. Phys. Rev. Lett. 36, 1084-1086 (1976).

2 Allen, P.B. Theory of resistivity "saturation". Superconductivity in $d$ - and f-Band Metals Eds. H. Suhl and M.B. Maple, Academic (New York,1980) p. 291-304.

${ }^{3}$ Hebard, A.F., Palstra, T.T.M., Haddon, R.C. \& Fleming, R.M. Absence of saturation in the normal-state resistivity of thin films of $\mathrm{K}_{3} \mathrm{C}_{60}$ and $\mathrm{Rb}_{3} \mathrm{C}_{60}$. Phys. Rev. B 48, 9945-9948 (1993).

${ }^{4}$ Degiorgi, L. et al., Optical response of the superconducting state of $\mathrm{K}_{3} \mathrm{C}_{60}$ and $\mathrm{Rb}_{3} \mathrm{C}_{60}$. Phys. Rev. Lett. 69, 2987-2990 (1992).

${ }^{5}$ Hou, J.G. et al., Determination of superconducting and normal state parameters of single crystal $\mathrm{K}_{3} \mathrm{C}_{60}$. Solid State Commun. 86, 643-646 (1993).

${ }^{6}$ Hou, J.G. et al., Resistivity saturation in alkali-doped $\mathrm{C}_{60}$. Solid State Commun. 93, 973-977 (1995).

${ }^{7}$ Gunnarsson, O. Superconductivity of Fullerides. Rev. Mod. Phys. 69, 575-606 (1997).

${ }^{8}$ Vareka, W.A. \& Zettl, A. Linear temperature dependent resistivity in $\mathrm{Rb}_{3} \mathrm{C}_{60}$. Phys. Rev. Lett. 72, 4121-4124 (1994).

${ }^{9}$ Satpathy, S. et al. Conduction-band structure of alkalidoped $\mathrm{C}_{60}$. Phys. Rev. B 46, 1773-1792 (1992).

${ }^{10}$ Mazin, I.I. et al. Orientational order in $\mathrm{A}_{3} \mathrm{C}_{60}$. Antiferromagnetic Ising model for the fcc lattice. Phys. Rev. Lett. 26, 4142-4145 (1993).

${ }^{11}$ Stephens, P.W. et al.. Structure of single-phase superconducting $\mathrm{K}_{3} \mathrm{C}_{60}$. Nature 351, 632-634 (1991).

12 Blankenbecler, R., Scalapino, D.J. \& Sugar, R.L. Monte Carlo calculations of coupled boson-fermion systems. I Phys. Rev. D 24, 2278-2286 (1981).

13 Jarrell, M. \& Gubernatis, J.E. Bayesian inference and the analytical continuation of imaginary-time quantum Monte Carlo data. Phys. Rep. 269, 133-195 (1996).

${ }^{14}$ Millis, A.J.,Hu, J. \& Das Sarma, S. Resistivity saturation revisited: Results from a dynamical mean field theory. Phys. Rev. Lett. 82, 2354-2357 (1999).

${ }^{15}$ Georges, A. et al. Dynamical mean-field theory of strongly correlated fermion systems and the limit of infinite dimensions. Rev. Mod. Phys. 68, 13-125 (1996).

${ }^{16}$ Holstein, T. Theory of transport phenomena in an electronphonon gas. Ann. Phys. 29, 410-535 (1964).

17 Pålsson, G. \& Kotliar, G. Thermoelectric response near the density driven Mott transition. Phys. Rev. Lett. 80, 4775-4778 (1998)).

${ }^{18}$ G. Grimvall, The Electron-Phonon Interaction in Metals, North-Holland (Amsterdam, 1981) p. 212.

${ }^{19}$ Gelfand,M.P. \& Lu, J.P. Orientational disorder and normal-state electrical-transport properties of $\mathrm{A}_{3} \mathrm{C}_{60}$. Phys. Rev. B 46, 4367-4370 (1992).

${ }^{20}$ Allen, P.B. \& Silberglitt, R. Some effects of phonon dynamics on electron lifetime, mass renormalization, and super- 
conducting transition temperature. Phys. Rev. B 9, 47334741 (1974).

\section{Acknowledgements}

We are grateful to M. Jarrell for making his MaxEnt program available and E. Koch for a careful reading of the manuscript. The work has been supported by the Max-Planck-Forschungspreis.

Correspondence and requests for materials should addressed to O.G.

(e-mail gunnar@and.mpi-stuttgart.mpg.de) 